Document downloaded from:

http://hdl.handle.net/10251/64818

This paper must be cited as:

Giner Sanz, JJ.; Ortega Navarro, EM.; Pérez-Herranz, V. (2015). Total harmonic distortion based method for linearity assessment in electrochemical systems in the context of EIS. Electrochimica Acta. 186:598-612. doi:10.1016/j.electacta.2015.10.152.

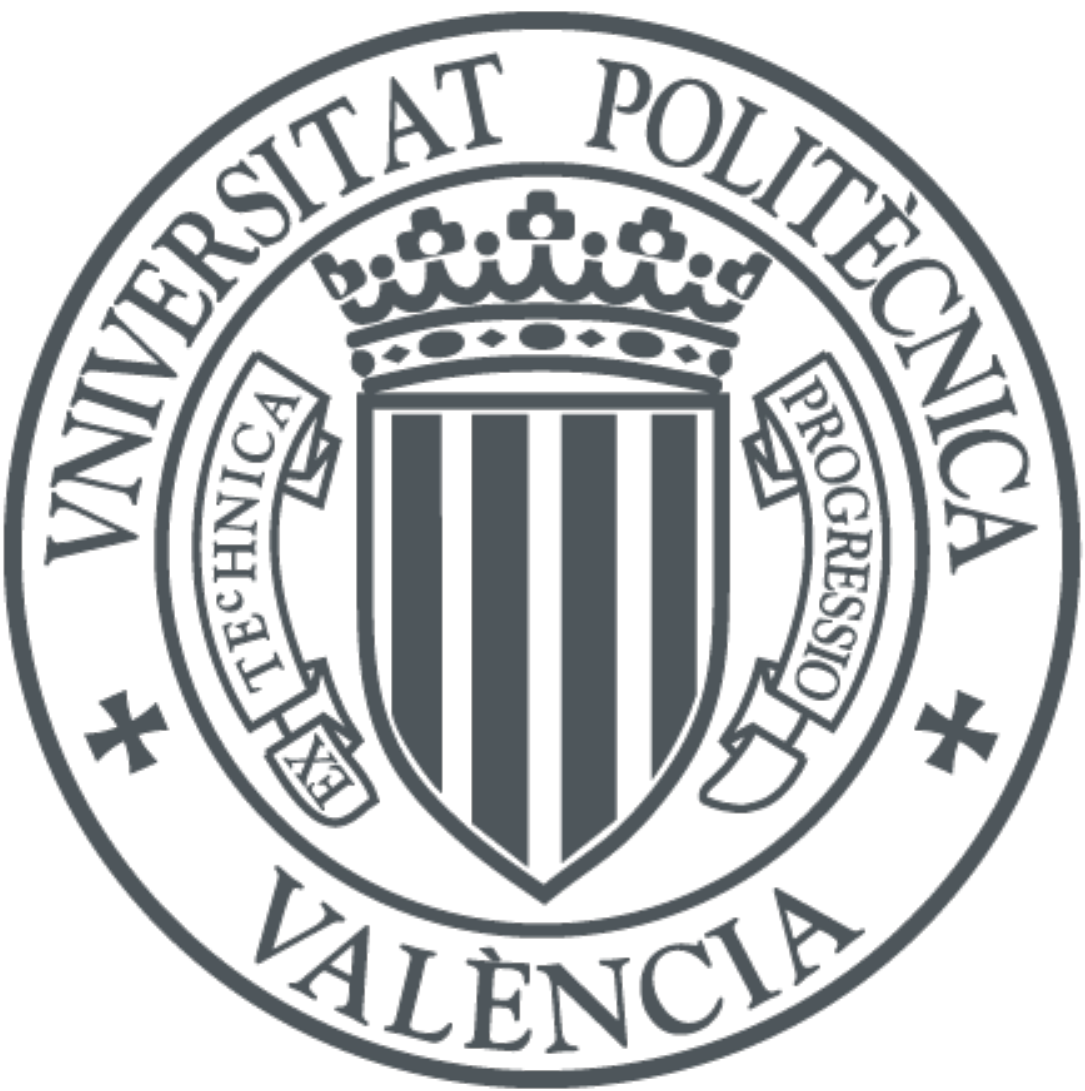

The final publication is available at

http://dx.doi.org/10.1016/j.electacta.2015.10.152

Copyright Elsevier

Additional Information 


\title{
Total harmonic distortion based method for linearity assessment in electrochemical systems in the context of EIS
}

\author{
J. J. Giner-Sanz, E. M. Ortega, V. Pérez-Herranz* \\ IEC group, Depto. Ingeniería Química y Nuclear, Universitat Politècnica de València \\ Camino de Vera S/N, 46022 Valencia, Spain \\ *Corresponding author. Tel.: +34-96-3877632; fax: +34-96-3877639; \\ E-mail address: vperez@iqn.upv.es (V. Pérez-Herranz)
}

\begin{abstract}
$\underline{\text { Abstract }}$
Electrochemical Impedance Spectroscopy (EIS) is a widely used electrochemical measurement technique that has been used in a great spectrum of fields since it allows deconvolving the individual physic-chemical processes that take place in a given system. Ohm's generalized law, and thus the impedance concept, are only valid if 4 conditions are fulfilled: causality, finiteness, stationarity and linearity. In the case that any of these conditions is not achieved, the obtained impedance spectra will present distortions that may lead to biased or even erroneous results and conclusions. For this reason it is crucial to verify if the 4 conditions are fulfilled, before accepting the results extracted from impedance spectra. In this work, a linearity assessment quantitative method based in the total harmonic distortion (THD) parameter is presented and verified experimentally. The experimental validation of the implemented method showed that the implemented method is able to assess quantitatively the linearity of the system. In addition, it is also able to determine the threshold frequency above which the system will not present significant nonlinear effects even for large perturbation amplitudes. It was observed that the THD method is more sensitive to nonlinear effects than the spectra themselves.
\end{abstract}

Keywords: Electrochemical Impedance Spectroscopy, Total harmonic distortion, linearity condition, spectrum validation, noise quantification. 


\section{Introduction}

In current days, electrochemical impedance spectroscopy (EIS) is one of the most used electrochemical techniques [1-4]. It has been applied to a wide range of different fields as fuel cells [5-10], batteries [11-12], coatings [13-14], electrochemical reaction kinetics [15], electrochemical sensors [16-19], supercapacitors [20-22] and dielectrics [23]. This electrochemical measurement technique has also been used in fields that are not traditionally linked to electrochemistry as enzymatic kinetics [24], cancer detection [25], biochemistry [26-27] and immunology [28-29] amongst others. The large spectrum of fields in which EIS has been applied is explained by the fact that this electrochemical method allows to deconvolve the different physic-chemical phenomena taking place in a given system [4]. This technique can be used both, in liquid systems [30-31] and solid systems [32-33].

The EIS technique consists in the application of a sinusoidal voltage (or current) perturbation to the system; and the measurement of the generated current (or voltage) output signal [1]. This process is repeated for different perturbation frequencies. For each frequency, the impedance of the system is determined, obtaining in this manner the EIS spectrum of the system. The impedance at angular frequency $\omega, Z(\omega)$, of a given system is defined by generalized Ohm's law [2]:

$$
Z(\omega)=\frac{\mathcal{F}[U(t)]}{\mathcal{F}[I(t)]}
$$

Where $\mathcal{F}$ stands for the Fourier transform operator; $U(t)$ denotes the potential signal in the time domain; and $I(t)$ is the current signal in the time domain. Therefore, the impedance concept is a generalization of the DC electric resistance concept: it quantifies not only the electric resistance of the system (amplitude relation between the current and voltage signals); but it also quantifies the time offset between both signals.

The complex Ohm's law, and thus the impedance concept, are only valid if the hypotheses of causality, finite range, linearity and stability are met [34]. If any of these conditions is not fulfilled the obtained spectra may be misleading and unusable to extract proper conclusions on the system.

A linear system is a system in which the relation between the perturbation and the response is a set of linear differential equations [35]. In other words, a linear system is a system where the superposition principle holds: the response to the sum of individual perturbations is the sum of the individual responses to each of these 
perturbations [36]. The nonlinearity of a system leads to the generation of harmonics, which distort the obtained EIS spectra [37].

However, the electrochemical systems are in general highly nonlinear systems since they are governed by Buttler-Volmer's equation [38]. Thus, in order to ensure compliance with the linearity condition in this kind of systems, the applied perturbation amplitude has to be small enough [39]. However, small amplitude perturbation signals lead to low signal-to-noise ratios [1]. Therefore, the quality of the measurement of impedance spectra in electrochemical systems is determined by a trade-off between the linearity of the system and the strength of the signal (signal-tonoise ratio) [40]. On the one hand, very low amplitude perturbations guarantee the linearity of the system but lead to large measurement errors due to a low signal-tonoise ratio. On the other hand, high amplitude perturbations lead to high signal-tonoise ratios, but they generate harmonics in the response signal due to the nonlinearity of the system. These harmonics introduce a systematic error in the impedance spectra measurement, due to the non-fulfilment of the linearity condition. Thus, the linearity assessment of an electrochemical system is essential for the determination of the optimum perturbation amplitude, and for the validation of impedance spectra measurements from a linearity point of view.

Commonly, the linearity of a system is experimentally verified using Lissajous figures [1]. This technique has two major drawbacks. On the one hand, a Lissajous figure is obtained for each frequency at which the impedance is measured. Therefore, each one of these Lissajous figures should be analyzed one by one: a typical impedance spectrum may require the analysis of around fifty Lissajous figures. As a consequence, a complete linearity study based on Lissajous figures requires a considerable amount of effort and time. On the other hand, the linearity assessment using Lissajous figures is a qualitative method: it allows detecting nonlinearities in a qualitative way, but does not allow quantifying the nonlinearity level. Furthermore, while the distortion of Lissajous figures associated to severe nonlinearities is easily recognizable; the distortion due to low and moderate nonlinearities is not so clear, and its identification can be subjected to the subjectivity of the person analysing the figure [41]. For these reasons, the use of Lissajous figures for linearity assessment should be reserved for an auxiliary experimental validation method only.

Another set of methods for evaluating the linearity of an electrochemical system is the set of methods based on harmonic analysis: the analysis of the system's response in the frequency domain [42]. The main advantage of this group of methods over the Lissajous method is that these methods are quantitative methods: they allow quantifying the nonlinearity level without being subject to the subjectivity of the person analysing the data. 
The aim of this work is to develop and experimentally validate a quantitative method for linearity evaluation in the context of electrochemical impedance spectroscopy, based on the analysis of the system's response in the frequency domain. The method should be able to quantitatively assess the linearity of the system, and to distinguish the nonlinear effects from the noise. The experimental validation of the method was done by applying the developed method to an alkaline hydrogen evolution electrochemical cell. 


\section{Harmonic analysis and total harmonic distortion}

When a mono-frequency sinusoidal perturbation signal is applied to a linear system, a mono-frequency response signal is obtained. This response signal has the same frequency that the perturbation signal. However, in the case of a nonlinear system, the response signal, when a mono-frequency sinusoidal perturbation is applied, is a multifrequency signal: the response signal will correspond to a superposition of different sine waves of different frequencies [43]. Therefore, if the linearity condition is not satisfied in electrochemical impedance measurements, the response signal will have sine waves of different frequencies superimposed to the fundamental signal, which frequency corresponds with the frequency of the perturbation signal. The frequencies of the superimposed signals correspond to integer multiples of the fundamental frequency: a nonlinear system generates non fundamental harmonics in the response signal [44].

There are a great number of theoretical works that demonstrate the generation of non-fundamental harmonics due to the nonlinearity of the system during electrochemical impedance measurements; such as the works of Darowicki [39], Diard and coworkers [45], Van Gheem and coworkers [46], and Victoria and Ramathan [37].

The harmonic generation due to the nonlinearity of the system is the basis of the linearity assessment methods based on harmonic analysis, selected for the present work. These methods are based in the analysis of the response signal in the frequency domain: The analysis of the harmonic content of the response signal of the system can be used to quantify the nonlinearity of the system.

Total harmonic distortion $(\mathcal{T H} \mathcal{H})$ is a parameter widely used to quantify the level of harmonics in current and voltage signals [47]. It is a widespread parameter in electrical engineering; and its most common use is the quality control of power supply: in fact this parameter appears in both national [48] and international [49] regulations concerning power supply.

Since the harmonic content of the response signal of the system can be used to quantify the nonlinearity of the system, the total harmonic distortion is a good parameter to quantify the nonlinearity of the system. For this reason, this parameter was selected for this work.

In literature, two different definitions of the total harmonic distortion can be found. On the one hand, one definition takes the fundamental component as reference. On 
the other hand, the other definition takes the root mean square (rms) value of the signal as reference. It has been shown that the first definition is a better measure of the harmonic content of an electrical signal [50]. Consequently, in this work the following definition of the total harmonic distortion of electrical signal $X$ was considered:

$$
\mathcal{J H} \mathcal{H} X=\frac{1}{|\widehat{X}|_{1}} \cdot \sqrt{\sum_{i=2}^{+\infty}|\widehat{X}|_{i}^{2}}
$$

Where $|\hat{X}|$ denotes the amplitude of the Fourier transform of signal $X$ (signal in the frequency domain). Subscript 1 stands for the fundamental component; while subscripts greater or equal to 2 are associated to non-fundamental harmonics.

Therefore, the total harmonic distortion of a current signal is given by:

$$
\mathcal{J H} \mathcal{D} I=\frac{1}{|\widehat{I}|_{1}} \cdot \sqrt{\sum_{i=2}^{+\infty}|\widehat{I}|_{i}^{2}}
$$

And the total harmonic distortion of a voltage signal is given by:

$$
\mathcal{T H \mathcal { D } U}=\frac{1}{|\widehat{U}|_{1}} \cdot \sqrt{\sum_{i=2}^{+\infty}|\widehat{U}|_{i}^{2}}
$$

These parameters were used in this work to quantify the level of harmonics in the perturbation and the response signals during EIS measurements; since, as it was explained, the level of harmonics quantified the nonlinearity of the system. 


\section{Methodology}

The linearity assessment method implemented in this work's frame consists in three main steps. Firstly, the impedance spectrum of the system is recorded as usual. But rather than saving only the impedance results, the current and voltage signals in the time domain are recorded for each excited frequency. The start point of the linearity assessment method is the raw signals in the time domain $(I(t)$ and $U(t))$ for each excited frequency. Secondly, using a Fast Fourier Transform (FFT) algorithm the signals in the time domain are transformed into the frequency domain: $\hat{I}(\vartheta)$ and $\widehat{U}(\vartheta)$, where $\vartheta$ represents the independent variable of the frequency domain. This transformation is performed for each excited frequency. Finally, once the signals have been transformed to the frequency domain, the total harmonic distortion of each signal can be determined using equations (3) and (4). Thus, the total harmonic distortion of the current signal $(\mathcal{T H} \mathcal{H} I)$ and of the voltage signal $(\mathcal{T H} \mathcal{H} U)$ is obtained for each excited frequency.

These results are analyzed using two tools: the $\mathcal{T H \mathcal { D }}$ curves and the critical parameters. On the one hand, the $\mathcal{T H} \mathcal{H}$ curves correspond to the curves of $\mathcal{T H} \mathcal{H} I$ and $\mathcal{T H} \mathcal{H} U$ versus the excited frequency. On the other hand, the critical frequency is defined as the excited frequency for which the total harmonic distortion values are maximal (highest harmonic content). Therefore, the critical $\mathcal{T H \mathcal { H }}$ parameters, $\mathcal{T H} \mathcal{H} I_{c}$ and $\mathcal{T H} \mathcal{H} U_{c}$, are defined by:

$$
\begin{aligned}
\mathcal{T H} \mathcal{H} I_{c} & =\max _{\mathrm{j} \in\left\{1 ; 2 ; \cdots ; N_{f}\right\}} \mathcal{T H \mathcal { D } I _ { j }} \\
\mathcal{T H} \mathcal{H} U_{c} & =\max _{\mathrm{j} \in\left\{1 ; 2 ; \cdots ; N_{f}\right\}} \mathcal{T H} \mathcal{H} U_{j}
\end{aligned}
$$

The critical parameters correspond to a high level analysis: they provide an overall assessment of linearity, since they consider the most unfavourable frequency (the frequency with the highest harmonic content). Whereas the $\mathcal{T H} \mathcal{H}$ curves correspond to a lower level analysis: they allow evaluating linearity for each frequency individually. Thus, these curves are very useful to distinguish for which frequencies the studied system is more susceptible to present nonlinearities.

The linearity assessment method is outlined in figure 1. This linearity assessment algorithm can be automated in any programming language. In this work, it was fully

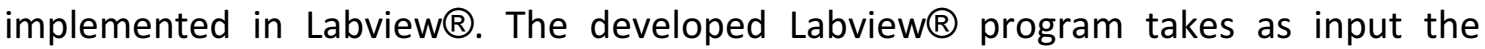
output of NOVA®, which is the software used to control the impedance measurement system, as detailed in the experimental work section. The NOVA®'s output consists in the raw signals (current and voltage) in the time domain. The implemented Labview $®$ program reads these raw signals in the time domain, calculates the associated signals 
in the frequency domain using an FFT algorithm; and once, the signals in the frequency domain have been obtained, it calculates the total harmonic distortion.

In order to validate the implemented method it is necessary to experimentally verify whether the method is able to identify and quantify nonlinearities, or not. The method should be validated in a large range of nonlinearities. Consequently, a highly nonlinear electrochemical system should be used for this experimental validation; since for highly nonlinear systems a slight increase in the amplitude of the perturbation produces a large increase in the nonlinearity of the system.

Using this criterion, a cathodic electrode of an alkaline electrolyser was selected to perform the experimental validation of the method, since it is a highly nonlinear electrochemical system. The system was described in detail by Herraiz-Cardona [51]. Due to its high nonlinearity, the selected system allows to obtain a wide range of nonlinearities, simply by varying the amplitude of the imposed disturbance. 


\section{Experimental work}

The experimental set up is shown in figure 2. This setup was described in detail by Herraiz-Cardona and co-workers [53-54]. The experiments were done using an electrochemical cell patented by the Dpto. Ingeniería Química y Nuclear of the Polytechnic University of Valencia [55]. It consists in a three-electrode cell with a heating circuit to control the temperature. The cell has two horizontal openings, and three vertical openings. The working electrode is placed in one of the horizontal positions; while the counter-electrode and the reference electrode are placed in two of the vertical openings. The third vertical opening acts as the gases outlet in order to prevent an overpressure inside the cell because of the volume of gases produced during the electrolysis. The main advantages of this type of electrochemical cell are: it minimizes de ohmic drop between the working electrode and the reference electrode; it allows the use of high surface area counter-electrodes; and it requires small volumes of electrolyte.

On the one hand, one of the electrodes developed by Herraiz-Cardona and co-workers was used as the working electrode. On the other hand, a nickel foam with very high surface area (Incofoam ${ }^{\circledR} 0.17 \mathrm{~cm}$ thick and 50 pores per linear inch) was used as counter-electrode. Finally, a commercial $\mathrm{Ag} / \mathrm{AgCl}(3 \mathrm{M} \mathrm{KCl})$ electrode was used as the reference electrode. The employed electrolyte was oxygen free $30 \mathrm{wt} . \% \mathrm{KOH}$ solutions. Before each experimental session, fresh electrolyte was prepared using $85 \mathrm{wt} . \% \mathrm{KOH}$ lentils (Panreac $($ ). To avoid carbonation of the solution, which would decrease its conductivity, the electrolyte was deaerated by bubbling $\mathrm{N}_{2}$ for 15 min prior to use. All experiments were performed at $30^{\circ} \mathrm{C}$ in galvanostatic mode, for a polarization current of $-10 \mathrm{~mA}$, since in preliminary works it was determined that it corresponded to the most nonlinear point of the system. As explained in the pervious section, a different perturbation amplitude was used in each experiment. 12 perturbation amplitudes were considered: $0.1 \mathrm{~mA}, 0.5 \mathrm{~mA}, 1 \mathrm{~mA}, 2 \mathrm{~mA}, \ldots ., 10 \mathrm{~mA}$

The measurements were performed using an Autolab $® 302 \mathrm{~N}$ potentiostat/galvanostat with FRA module, controlled using NOVA $®$ software. The selected frequency range was $10 \mathrm{kHz}-5 \mathrm{mHz}$, with 10 frequencies per decade. The used measurement parameters are listed in table 1.

Before each experiment, a pre-treatment was applied to the working electrode in order to reduce any oxides that could have been generated on its surface; and thus have similar surface conditions in all experiments. The applied pre-treatment consisted in applying a $-1.6 \mathrm{~V}$ potential during 30 minutes. 


\section{Results and discussion}

\section{$\underline{\text { 5.1. Nyquist plots }}$}

Figure 3 shows the Nyquist plots obtained for 4 selected perturbation amplitudes: 0.1 $\mathrm{mA}, 2 \mathrm{~mA}, 6 \mathrm{~mA}$ and $10 \mathrm{~mA}$. All the spectra consist in a displaced depressed capacitive semicircle, formed by two overlapping semi-circles, one for high frequencies and the other one for intermediate frequencies [52]. The physical explanation of these spectra was widely described by Herraiz-Cardona et al. [51, 52-54]. The intermediate frequency semi-circle is related to the Hydrogen Evolution Reaction (HER) kinetics; while the high frequency semi-circle is related to the porosity of the electrode surface. Finally, the displacement of the semicircle with respect to the origin is associated to the electrolyte resistance.

Although the general shape of the spectrum is the same for the different perturbation amplitudes, a significant variation of the spectra with the perturbation amplitude can be observed in figure 3 .

On the one hand, comparing the spectra obtained for low amplitudes $(0.1 \mathrm{~mA}$ and 2 $\mathrm{mA}$ ) it can be observed that they are almost identical, and the main discrepancy is in the low frequency zone. This variation of the spectra obtained with low perturbation amplitudes for low frequencies is not due to nonlinear effects, but it is due to the variability of the measurements at low frequencies. For the spectra obtained with a 0.1 $\mathrm{mA}$ perturbation the variability of the measurement for low frequencies is much higher than in the case of the $2 \mathrm{~mA}$ perturbation. This is due to the fact that in the 0.1 $\mathrm{mA}$ case, the signal-to-noise ratio is very low. This causes the noisy pattern in the low frequency zone observed in the $0.1 \mathrm{~mA}$ case.

On the other hand, no noisy pattern is observed in the $2 \mathrm{~mA}, 6 \mathrm{~mA}$ and $10 \mathrm{~mA}$ cases. Instead, a systematic distortion is observed: an increase of the perturbation amplitude causes a significant expansion of the intermediate frequency capacitive semicircle. This distortion is due to the emergence of nonlinear effects for large perturbation amplitudes.

Moreover, in figure 3, it can be observed that the spectrum distortion is only observed for low/intermediate frequency; whereas for high frequency no significant difference can be appreciated between the 4 spectra. This shows that the nonlinear effects only appear under a threshold frequency. For frequencies higher than the threshold the nonlinear effects are neglectable even for very large amplitudes: there will be no distortion of the spectrum in the frequency zone corresponding to frequencies larger than the threshold frequency. On the contrary, for frequencies below the threshold 
the nonlinear effects are not neglectable above a critical amplitude of the perturbation: for amplitudes above the critical amplitude, a significant distortion in the frequency zone corresponding to frequencies below the threshold frequency will be observed. The existence of a frequency threshold above which nonlinearities are neglectable was already observed by Hirschorn and Orazem [56].

\subsection{AC signals and Lissajous plots}

The common practice to identify nonlinear behaviour is to use AC plots and Lissajous figures [1]. For this reason, in this section, the AC plots and the Lissajous figures obtained experimentally in this work are presented and discussed.

On the one hand, figure 4 shows the AC signals (current and voltage) in the time domain for three selected frequencies from different frequency zones of the Nyquist plot ( $100 \mathrm{~Hz}, 1 \mathrm{~Hz}$ and $5 \mathrm{mHz}$ ); for two perturbation amplitudes ( $1 \mathrm{~mA}$ and $10 \mathrm{~mA}$ ). The selected frequencies are labelled in the Nyquist plot shown in figure 3 as $A(100 \mathrm{~Hz}), B$ $(1 \mathrm{~Hz})$ and $\mathrm{C}(5 \mathrm{mHz})$. For a given frequency, it can be observed that the perturbation signals (current signals) for both amplitudes have the same shape and frequency, only the amplitude of the signal varies from one to the other. Therefore, the linearity of the system does not affect the perturbation signals. It can be deduced from this observation that there is no back resonance in the system that would introduce harmonics in the perturbation signal due to nonlinear effects. This observation is consistent with the causality assumption. In the case of $1 \mathrm{~mA}$, for a given frequency, it can be observed that the output signal (voltage) has the same shape and frequency than the perturbation signal, with different amplitude and a given offset. According to generalized Ohm's law the amplitude ratio and the offset define the impedance of the system at that frequency. The fact that both signals (input and output) for a given frequency and a perturbation amplitude of $1 \mathrm{~mA}$ have the same frequency indicates that the system behaves as a linear system for a perturbation amplitude of $1 \mathrm{~mA}$. In contrast, this is not true for the three considered frequencies in the $10 \mathrm{~mA}$ case: for $100 \mathrm{~Hz}$, the output signal is a sinusoidal signal of same frequency as the perturbation signal; but for $1 \mathrm{~Hz}$ and $5 \mathrm{mHz}$, the output signal is not a mono-frequency sinusoidal signal. For $1 \mathrm{~Hz}$ and $5 \mathrm{mHz}$ the output signal corresponds with a combination of sine signals of different frequencies, producing the distorted signals observed in figure 4. This shows that the system has a significant nonlinear behaviour for a perturbation amplitude of $10 \mathrm{~mA}$ at $1 \mathrm{~Hz}$ and $5 \mathrm{mHz}$.

On the other hand, figure 5 represents the Lissajous figures corresponding to the pairs of signals shown in figure 4. Lissajous figures allow a better and faster visualization of the information contained in the AC signals [1]. For the $1 \mathrm{~mA}$ case, it can be observed that the Lissajous figures obtained for the three selected frequencies are symmetric 
with respect to the origin. From this observation it can be deduced that both signals (input and output) are mono-frequency sine signals of same frequency [57]; thus, the system behaves linearly for a perturbation amplitude of $1 \mathrm{~mA}$ [2]. On the contrary, for a $10 \mathrm{~mA}$ perturbation a clear distortion in Lissajous figures can be observed for $1 \mathrm{~Hz}$ and $5 \mathrm{mHz}$. This type of distortion is associated with the presence of harmonics in the signal, and thus to nonlinear effects. So, as from the analysis of the AC plots, from the Lissajous figures it can also be deduced that the system behaves nonlinearly at $1 \mathrm{~Hz}$ and $5 \mathrm{mHz}$ for an amplitude of $10 \mathrm{~mA}$.

The nonlinear behaviour only was detected for high amplitude and low frequencies. Even for high amplitudes, the system does not behave nonlinearly for high frequencies. This observation shows that the nonlinear effects only appear under a threshold frequency. This is consistent with the observations presented in section 5.1: On the one hand, for frequencies higher than the threshold the nonlinear effects are neglectable even for very large amplitudes: there will be no distortion of the spectrum. Thus this part of the spectrum can be used with no fear of introducing bias in the results of the analysis because of a distortion due to nonlinear effects. On the other hand, for frequencies below the threshold the nonlinear effects are not neglectable above a critical amplitude of the perturbation. Consequently, this part of the spectrum may introduce a bias in the results of the analysis because of the distortion due to nonlinear effects in the case that the used amplitude is above the critical amplitude.

The problem of using this type of linearity assessment method is that it requires a big effort in case that the analyst wants to determine the threshold frequency. In case that the analyst only wants to know if the linearity condition can be accepted or not for the whole spectrum, then only the lowest frequency Lissajous plot should be analysed (since nonlinear effects appear at frequencies lower than the threshold frequency). However, if the analyst wants to determine the threshold frequency, then all the frequencies should be analysed. In this case, only three of the 64 frequencies at which the impedance was recorded were analysed. A full linearity analysis would require analyzing one by one the 64 frequencies of each spectrum. Therefore, this kind of approach would be very time consuming. But there is a bigger drawback of this Lissajous based linearity assessment method than the large amount of work it requires: it is a qualitative method. In this section, only the extreme cases have been presented. For these extreme cases the distortion is very clear. However, for the boundary cases the distortion is not so easily recognizable; and the detection of nonlinearities may be subjected to certain subjectivity of the analyst. In contrast, the method presented in this work is a quantitative method. This is the major advantage of the method presented in this work in comparison with the Lissajous method, which is the method commonly used to experimentally verify the linearity condition [1]. 


\subsection{THD curves}

Figures 6 and 7 show the $\mathcal{T H} \mathcal{H}$ curves for the perturbation signal and for the output signal respectively. Each figure is divided in two parts: one for low perturbation amplitudes (a) and another one for high perturbation amplitudes (b). These curves consist in the representation of the $\mathcal{T H \mathcal { D }}$ value, determined using equations (3) and (4), versus the perturbation signal frequency.

On the one hand, in figure 6 it can be observed that the $\mathcal{T H} \mathcal{D}$ curves for the perturbation signal $(\mathcal{T H} \mathcal{H} I)$ present the same pattern for all perturbation amplitudes. For a given perturbation amplitude, the $\mathcal{T H} \mathcal{H} \mathcal{D} I$ parameter is approximately constant for high frequencies. A $\mathcal{T H} \mathcal{H} I$ peak is observed for intermediate frequencies (in the range $10 \mathrm{~Hz}-0.1 \mathrm{~Hz}$ ). And finally, the $\mathcal{T H} \mathcal{H} \mathcal{D} I$ parameter stabilizes for low frequencies. In figure 6.a it can be observed that for low perturbation amplitudes an increase of the amplitude shifts the $\mathcal{T H} \mathcal{H} I$ curve to lower values. In contrast, as it can be observed in figure 6.b, for amplitudes larger than $2 \mathrm{~mA}$ an increase in the amplitude has no significant effect on the $\mathcal{T H} \mathcal{H} I$ curve. Therefore, an increase of the perturbation amplitude causes a drop of the $\mathcal{T H} \mathcal{H} I$ parameter for every frequency for low amplitudes; while for amplitudes higher than a threshold amplitude, the $\mathcal{T H} \mathcal{H} I$ curves do not show a clear trend, and can be considered as nearly superimposed. These $\mathcal{T H} \mathcal{H} I$ curves are characteristic of the measurement equipment, which is responsible of generating the perturbation and applying it to the system. These curves can be affected by distortions in the grid signal that powers the potentiostat/galvanostat, and by electric couplings between the measurement equipment and other electric and electronic devices connected to the grid. For low amplitudes, an increase in the perturbation amplitudes leads to an improvement of the signal-to-noise ratio. This improvement causes a decrease of the $\mathcal{T H} \mathcal{H} \mathcal{D} I$ parameter (a lower harmonic relative level with respect to the signal) as observed in figure 6.a. However, there is an amplitude (of around $2 \mathrm{~mA}$, in this case) above which further increases of the amplitude do not improve the signal-to-noise ratio anymore, since the limit of the measurement system (potentiostat/galvanostat, electric network, etc...) has been reached. Beyond this point the $\mathcal{T H} \mathcal{H} \mathcal{D} I$ parameter does not decrease further, resulting

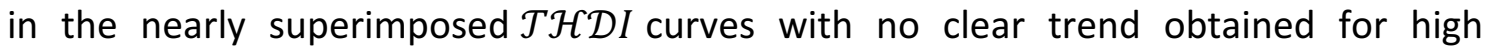
amplitudes, as seen in figure 6.b.

The highest $\mathcal{T H} \mathcal{H} I$ value corresponds to the intermediate frequency peak of the curve of $0.1 \mathrm{~mA}$ (which has associated the larger values of $\mathcal{T H} \mathcal{H} I$, as discussed previously). This maximum $\mathcal{T} \mathcal{H} \mathcal{D} I$ value is under $2 \%$. Therefore, the $\mathcal{T} \mathcal{H} \mathcal{D} I$ parameter is under $2 \%$ for every frequency and every amplitude considered in this work. The $\mathcal{T H \mathcal { H }}$ limit in general distribution networks is $8 \%$ [48]. Consequently, in this case, the $\mathcal{T H} \mathcal{H} I$ value for all frequencies and amplitudes is below this reference limit: it can be deduced that 
even for the lower amplitude, the signal-to-noise ratio of the perturbation imposed to the system is acceptable.

On the other side, it can be observed in figure 7 that the overall shape of the $\mathcal{T H} \mathcal{H}$ curves for the response signal $(\mathcal{T H} \mathcal{H} U)$ changes from low perturbation amplitudes (figure 7.a) to high amplitudes (figure 7.b). For low amplitudes (up to $2 \mathrm{~mA}$ ) the $\mathcal{T H} \mathcal{H} U$ curve is a point cloud with no appreciable trend or shape, with significantly higher values in the frequency range $100 \mathrm{~Hz}$ to $1 \mathrm{~Hz}$. This frequency range corresponds with the frequency range at which the peak was observed for the perturbation signal, as it was discussed above. It is further noted that an increase in the perturbation amplitudes for amplitudes below $2 \mathrm{~mA}$ causes a shift of the point cloud towards lower $\mathcal{T H} \mathcal{H} U$ values. This is due to the improvement of the signal-to-noise ratio: an increase in the amplitude of the disturbance signal, without exceeding the threshold amplitude above which the system can no longer be considered as linear, leads to an increase in the signal-to-noise ratio and this causes a decrease of the $\mathcal{T H} \mathcal{H} U$ parameter.

For high amplitudes (above $2 \mathrm{~mA}$ ) the $\mathcal{T H} \mathcal{H} \mathcal{D} U$ curves present a common pattern. For a given amplitude, three distinct zones can be identified in the $\mathcal{T H} \mathcal{H} U$ curve:

- For high frequencies, the $\mathcal{T H} \mathcal{H} \mathcal{D}$ curve is constant. In this section, the $\mathcal{T H} \mathcal{H} U$ values are very low. In this zone, two local peaks are observed for $50 \mathrm{~Hz}$ and 25 $\mathrm{Hz}$. Probably, these peaks are due to the coupling of the system with the grid;

- For midrange frequencies, the $\mathcal{T H} \mathcal{H} \mathcal{D} U$ curve is monotonically increasing, and presents an inflexion point;

- Finally, the curve shows an asymptotic behavior for low frequencies.

For high amplitudes, comparing the $\mathcal{T H} \mathcal{H} \mathcal{D}$ curves for different amplitudes it is observed that the $\mathcal{T H} \mathcal{H}$ U curves shift to higher values with an increase in amplitude. This is due to the nonlinear effects of the system: the harmonic generation due to nonlinear effects. For high amplitudes the nonlinear effects of the system are no longer neglectable: an increase in the amplitude causes greater nonlinear effects, and this causes an increase of the amplitude of the non fundamental harmonics generated due to the nonlinearity of the system, especially for low frequencies. This is consistent with the qualitative observations extracted from the Lissajous' figures in section 5.2.

In short, an increase in the perturbation amplitude for low amplitudes (lower than an amplitude threshold) causes a shift of the $\mathcal{T H} \mathcal{H} U$ curve towards lower values. For these low amplitudes, the $\mathcal{T H} \mathcal{H} \mathcal{D}$ curves have no defined shape, and consist in scattered point clouds. This drop of the $\mathcal{T H} \mathcal{H} U$ value with the amplitude, for low amplitudes, is due to the improvement of the signal-to-noise ratio. For low amplitudes, the non fundamental harmonics generated due to nonlinear effects of the system are neglectable. In this amplitude range, the noise dominates over the nonlinear effects. 
And therefore, the net effect is a decrease of the $\mathcal{T H} \mathcal{H} U$ value. From a certain amplitude threshold, a clear shape appears in the $\mathcal{T H} \mathcal{H} \mathcal{D}$ curve. Furthermore, for amplitudes above this amplitude threshold an increase in the perturbation amplitude causes a shift of the the $\mathcal{T H} \mathcal{H} \mathcal{D U}$ curve towards higher values. This is due to the fact that in this amplitude range, the nonlinear effects dominate over the noise: an increase in the amplitude causes the generation of non fundamental harmonics due to non linear effects that are not neglectable with respect to the noise. Therefore, the net effect is an increase of the non fundamental harmonic content of the output signal, and thus an increase of the $\mathcal{T H} \mathcal{H} \mathcal{D} U$ value.

For amplitudes larger than $2 \mathrm{~mA}$, the highest values of $\mathcal{T H} \mathcal{H} U$ for a given amplitude correspond to the low frequency zone: even for high amplitudes, the nonlinear effects are negligible for high frequencies (higher than $10 \mathrm{~Hz}$ ). This is consistent with the observations extracted from the Nyquist plots analysis: only the low frequency zone of the spectra is significantly distorted by the nonlinear effects; while the high frequency region is not altered noticeably. It can be deduced that this system presents a frequency threshold. This concept was introduced by Orazem and co-workers [58], and has already been discussed in the previous section. In this case, the frequency threshold is around $10 \mathrm{~Hz}$ : for frequencies above this threshold frequency, the system does not present nonlinear effects even for high perturbation amplitudes.

Comparing figures 6 and 7 , it is observed that the $\mathcal{T H} \mathcal{H}$ values associated to the output signal are much larger than the values associated to the input signal. It can be deduced that the major part of the harmonics present in the output signal (both, due to noise and to nonlinear effects) are generated by the system itself; since they are only present in the output signal. In addition, it can be concluded that the harmonic generation due to nonlinear effect only occurs in the output signal; there is no back resonance that could introduce harmonics in the perturbation signal due to electrical coupling between the measurement system and measured system. Therefore, in order to verify the linearity of a system only the output signal is relevant. However, to monitor the input signal is interesting in order to control the quality of the perturbation signal and to detect possible distortions in the grid that could introduce noise in the measurement.

It should be noted that if the EIS measurements were done in potenciostatic mode, rather than in galvanostatic mode, the results would be reversed: the total harmonic distortion of the current signal will be significantly higher than the total harmonic distortion in the voltage signal. Thus, in potenciostatic mode, the $\mathcal{T H} \mathcal{H}$ values of the potential signal will be neglectable; and the $\mathcal{T H \mathcal { D }}$ values of the current signal will contain the information of the nonlinearity effects and the noise produced by the system. 


\subsection{Critical parameter curves}

Figures 8 and 9 show the critical parameter curves for the perturbation signal and the response signal respectively. These curves correspond with the representation of the critical parameter $\left(\mathcal{T H} \mathcal{H} I_{c}\right.$ and $\left.\mathcal{T H} \mathcal{H} U_{c}\right)$ versus the amplitude of the perturbation. Note the different scales of figures 8 and 9: the $\mathcal{T H} \mathcal{H} I_{c}$ values are one order of magnitude lower than the values of $\mathcal{T H} \mathcal{H} U_{c}$ for all amplitudes. This is due to the fact that the system introduces additional noise, and for high perturbation amplitudes it also introduces non fundamental harmonics due to nonlinear effects. The result is that the critical parameter associated with the perturbation signal is significantly below the critical parameter associated with the output signal for a given amplitude. This is consistent with the observation stated in section 5.3 according to which the nonlinear effects only appear in the output signal and not in the perturbation.

On the one hand, in figure 8 it can be observed that the critical parameter associated with the perturbation signal decreases with the amplitude for very low amplitudes; and stabilizes around a value of around $0.8 \%$, for perturbation amplitudes larger than 1 $\mathrm{mA}$. When the perturbation amplitude is increased the signal-to-noise ratio of the perturbation signal improves, resulting in a drop of the critical parameter $\mathcal{T} \mathcal{H} \mathcal{D} I_{c}$. Once the limit of the measurement equipment that produces the perturbation signal is reached further increases in the amplitude do not improve further the signal-to-noise ratio. Thus, the parameter $\mathcal{T H} \mathcal{H} \mathcal{D} I_{c}$ remains approximately constant beyond this point.

On the other hand, in figure 9 two different trends are observed. For low amplitudes, an increase in the amplitude causes a decrease in the critical parameter of the output signal, $\mathcal{T H} \mathcal{H} U_{c}$, whereas at medium and high amplitudes, an increase in the amplitude leads to an increase of parameter $\mathcal{T H} \mathcal{H} U_{c}$. Therefore, a threshold amplitude can be identified as the amplitude at which the trend of $\mathcal{T} \mathcal{H} \mathcal{D} U_{c}$ inverts. An increase in the perturbation causes two antagonistic effects. On one side, it improves the signal-to-noise ratio, which causes a drop in $\mathcal{T H} \mathcal{H} U_{c}$. On the other side, it generates higher levels of non fundamental harmonics due to the nonlinear behavior of the system. For amplitudes below the threshold amplitude, the first effect dominates over the second one: the harmonic generation due to nonlinearity is neglectable compared with the improvement of the signal-to-noise ratio. Therefore, the net effect of an increase of the amplitude of the perturbation for low amplitudes is a drop in the critical parameter. Consequently, the system can be considered to behave linearly in this amplitude range. In contrast, for amplitudes above the threshold amplitude, the second effect dominates over the first one: the harmonic generation due to nonlinear effects is no longer neglectable compared to the signal-tonoise ratio improvement. Therefore, the net effect of an increase of the amplitude of the perturbation in this amplitude range is an increase of the critical parameter. 
Consequently, the nonlinear behavior of the system is significant for amplitudes above the amplitude threshold.

In short, the threshold amplitude identified in the $\mathcal{T H} \mathcal{H} U_{c}$ curve determine whether a system can be considered linear or not for a given amplitude. For amplitudes below the threshold, the nonlinear effects are neglectable; and consequently, the linearity hypothesis can be accepted. For amplitudes above the threshold, the nonlinear effects are no longer neglectable; and therefore the linearity hypothesis cannot be accepted. In this case, the obtained impedance spectra may be distorted, and lead to biased results. This threshold amplitude for the studied system can be extracted from figure 9: in this case, it is around $2 \mathrm{~mA}$. It can be deduced that in figure 3 , the spectrum obtained with a perturbation of $2 \mathrm{~mA}$ is the optimum spectrum (best signal-to-noise ratio without significant nonlinear effects): it is the spectrum with less error with respect to the "real" spectrum of the system.

\subsection{Noise quantification}

As it was observed in section 5.3, the input signal can be considered as a monofrequency senoidal signal with neglectable amount of noise. Therefore, it can be written as:

$$
I(t)=I_{D C}+\Delta I \cdot \sin (\omega t)
$$

Where $I_{D C}$ denotes the polarization current. This signal is applied to the system; and the output signal consists in the superposition of a fundamental signal and its harmonics. Each one of these signals is composed of two components: one due to the response of the system ( $\Delta U_{1}$ for its linear response; and $\Delta U_{k}$ (for $k \geq 2$ ) for its nonlinear response) and one associated to noise. Therefore, the output signal can be expressed as:

$$
U(t)=U_{D C}+\left(\Delta U_{1}+\Delta U_{n 1}\right) \cdot \sin \left(\omega t+\varphi_{1}\right)+\sum_{k=2}^{+\infty}\left(\Delta U_{k}+\Delta U_{n k}\right) \cdot \sin \left(k \omega t+\varphi_{k}\right)
$$

Where $U_{D C}$ stands for the DC component of the voltage signal. On the one hand, $\Delta U_{1}$ denotes the linear response of the system and $\Delta U_{n 1}$ is the noise in the fundamental component of the output signal. On the other hand, $\Delta U_{k}$ and $\Delta U_{n k}$ (for $k \geq 2$ ) denote the system's response and the noise in the k-th harmonic. Finally, $\varphi_{k}$ (for $k \geq 1$ ) is the offset of the k-th component of the output signal with respect to the input signal. 
In the linear behavior zone of the system (amplitudes lower than the threshold amplitude identified in section 5.4), the nonlinear effects of the system are neglectable with respect to the corresponding noise. Therefore, in this region:

$$
\Delta U_{k} \ll \Delta U_{n k} \forall k \in \mathbb{N} \mid k \neq 1
$$

Moreover, it can be considered that the fundamental component of the output signal is high enough to overcome the noise associated to it; if not, the measurement would be only noise. Therefore, it can be considered that:

$$
\Delta U_{1} \gg \Delta U_{n 1}
$$

Introducing assumptions (9) and (10) in equation (8), and particularizing the obtained expression for the critical frequency (since equation (8) can be applied to any measured frequency), the following expression is obtained:

$$
U(t)=U_{D C}+\Delta U_{1} \cdot \sin \left(\omega_{c} t+\varphi_{1}\right)+\sum_{k=2}^{+\infty} \Delta U_{n k} \cdot \sin \left(k \omega_{c} t+\varphi_{k}\right)
$$

Where $\omega_{c}=2 \pi f_{c}$ is the angular frequency associated to the critical frequency. Using the definition of the Fourrier transform:

$$
\begin{gathered}
|\widehat{U}|_{1}=\Delta U_{1} \\
|\widehat{U}|_{k}=\Delta U_{n k} \quad \forall k \in \mathbb{N} \mid k \neq 1
\end{gathered}
$$

Introducing these terms in the definition of $\mathcal{T H} \mathcal{H} U$, and since the critical frequency is being considered, the following expression for $\mathcal{T H} \mathcal{H} U_{c}$ is obtained for the linear zone:

$$
\mathcal{J H} \mathcal{D} U_{c}=\frac{1}{\Delta U_{1}} \cdot \sqrt{\sum_{k=2}^{+\infty}\left(\Delta U_{n k}\right)^{2}}
$$

According to Ohm's generalized law, the linear component of the system's response is given by: 


$$
\Delta U_{1}=\left|Z\left(f_{c}\right)\right| \cdot \Delta I
$$

Where $\left|Z\left(f_{c}\right)\right|$ denotes the modulus of the impedance of the system at the critical frequency.

Introducing expression (15) in (14), the following expression is obtained:

$$
\mathcal{T H} \mathcal{H} U_{c}=\frac{\lambda}{\Delta I}
$$

Where:

$$
\lambda=\frac{1}{\left|Z\left(f_{c}\right)\right|} \cdot \sqrt{\sum_{k=2}^{+\infty}\left(\Delta U_{n k}\right)^{2}}
$$

Parameter $\lambda$ has dimension of current. The following parameter can be defined:

$$
\chi=\sqrt{\sum_{k=2}^{+\infty}\left(\Delta U_{n k}\right)^{2}}
$$

This parameter (with dimension of voltage) is a measure of the total noise in the measurement. Therefore, in the linear zone of the $\mathcal{T H} \mathcal{H} U_{c}$ curve, the following relation holds:

$$
\mathcal{T H \mathcal { H }} U_{c}=\frac{\chi}{\left|Z\left(f_{c}\right)\right|} \cdot \frac{1}{\Delta I}
$$

This model was fitted to the linear zone of the experimental $\mathcal{T H} \mathcal{H} U_{c}$ curve (perturbation amplitudes below $3 \mathrm{~mA}$ ), using a Levenberg-Marquardt algorithm. The fitted model is shown in figure 11. It can be observed that the model given by equation (16) fits perfectly the experimental $\mathcal{T H} \mathcal{H} \mathcal{D} U_{c}$ curve in the linear zone: the associated determination coefficient is $R^{2}=99.998 \%$. It can be deduced that the proposed expression actually explains the experimental shape of the $\mathcal{T} \mathcal{H} \mathcal{D} U_{c}$ curve in the linear region. From the experimental fit, the value of parameter $\lambda$ can be obtained:

$$
\lambda=\frac{\chi}{\left|Z\left(f_{c}\right)\right|}=10.43 \mathrm{~A}
$$


In this case, the modulus of the impedance of the system at the critical frequency is $4.76 \Omega$. Thus, in this case: $\chi \approx 49.65 \mathrm{~V}$. This value quantifies the total noise in the measurement. Therefore, the noise can be quantified from the linear behavior zone of the THD critical curve.

\subsection{Nonlinear effects quantification}

The critical parameter can be decomposed in two contributions. One associated to the nonlinear effects of the system, and another one associated to the noise:

$$
\mathcal{T H} \mathcal{H} U_{c}=\mathcal{T} \mathcal{H} \mathcal{D} U_{c_{-} \text {nonlinear }}+\mathcal{T} \mathcal{H} \mathcal{D} U_{c_{-} \text {noise }}
$$

By definition, in the linear zone the nonlinear effects are neglectable. In addition, as it was developed in section 5.5, equation (16) gives the model of the critical parameter in the linear zone. Thus, it can be deduced that the component associated to noise (the only significant component in the linear zone) can be expressed as:

$$
\mathcal{T H} \mathcal{H} U_{c_{-} \text {noise }}=\frac{\lambda}{\Delta I}
$$

Where $\lambda$ is the parameter obtained from fitting the linear zone of the curve to the model developed in section 5.5.

Introducing (20) in (19) and rearranging terms, the following expression is obtained:

$$
\mathcal{T H} \mathcal{H} U_{c_{-} \text {nonlinear }}=\mathcal{T} \mathcal{H} \mathcal{D} U_{c}-\frac{\lambda}{\Delta I}
$$

Using this expression, the nonlinear effect contribution to the critical parameter can be calculated for each perturbation amplitude. The obtained values are shown in figure 12. It can be observed that in the linear zone (amplitudes lower or equal to $2 \mathrm{~mA}$ ) the nonlinear contribution is nearly zero; while in the nonlinear zone, the nonlinear contribution to the critical parameter increases nonlinearly with the perturbation amplitude. This is logical: on the one hand, in the linear zone the nonlinear effects are neglectable. On the other hand, in the nonlinear zone, greater perturbation amplitudes cause the generation of higher nonlinear effects.

The advantage of this method is that it is able to distinguish the nonlinear effects of the system from the noise; allowing for instance to compare the nonlinearity of two different systems. This is a major advantage of the described method with respect to other harmonic analysis that can be found in literature, that are not able to distinguish 
the nonlinear effects from the noise. Consequently, the method described in this work has a clear utility in the linearity assessment of electrochemical systems, and in the noise quantification and characterization. However, in literature, the total harmonic distortion has been used to obtain relevant information about the system itself. For instance, Mao and coworkers used total harmonic distortion to obtain relevant information about direct methanol fuel cells [59-61]. Further work should be done in order to determine if the method presented in this work can be used to obtain relevant information about a given system, other than linearity information, and noise quantification and characterization. 


\section{$\underline{\text { 6. Conclusions }}$}

The implemented method has successfully been validated from an experimental point of view: it is able to assess the linearity of the system in a quantitative way. The main advantage of this method over the methods commonly used for linearity assessment (i.e. Lissajous figures) is that the $\mathcal{T H \mathcal { H }}$ method is a quantitative method. Consequently, this method allows to accurately quantify both, the importance of the nonlinear effects of the system and the signal-to-noise ratio. Moreover, it is a quick method that does not require the one-by-one visual inspection of Lissajous figures in search of distortions due to nonlinear effects (that may not even be evident).

The method is able to determine the threshold amplitude which delimits the zone where the linearity condition can be accepted and the where it cannot be accepted. This may be used for optimizing the perturbation amplitude for a given system. The method also allows identifying the threshold frequency, above which no nonlinear significant effects appear even for high frequencies: this can be used to determine which part of the spectra is sensitive to nonlinear effects. Only this zone may present distortions due to nonlinearity. In the linear zone, the method allows quantifying the noise of the system; and it may even be used to track the sources of noise in the system (i.e. electric coupling with the grid).

In summary, the presented method allows to define the linear and the nonlinear zones of the system; obtain the optimum perturbation amplitude and the threshold frequency of the system; discriminate the nonlinear effects from the noise; and quantify and characterize the noise in the system. Moreover, the computational requirements of this algorithm are significantly low. This corresponds with the major advantage of this method with respect to other harmonic analysis methods: it allows obtaining all the linearity related parameters (optimum perturbation amplitude and threshold frequency) and to quantify and characterize the noise in the system with a single method that is based in an algorithm with relatively low computational requirements. 


\section{Nomenclature}

\section{Normal letters}

$\mathcal{F} \quad$ Fourier transform operator

$f \quad$ Frequency $(\mathrm{Hz})$

$f_{c} \quad$ Critical frequency $(\mathrm{Hz})$

I Current in the time domain $(A)$

$\hat{I} \quad$ Current in the frequency domain $(A)$

$I_{D C} \quad$ Polarization current $(A)$

$N_{f} \quad$ Number of measured frequencies

$t \quad$ Time domain independent variable $(s)$

$\mathcal{T H} \mathcal{D} X \quad$ Total harmonic distortion of signal $X(\%)$

$\mathcal{T H} \mathcal{H} X_{c} \quad$ Critical total harmonic distortion of signal $X$ (\%)

$U \quad$ Potential in the time domain $(V)$

$\widehat{U} \quad$ Potential in the frequency domain $(V)$

$Z \quad$ Complex impedance $(\Omega)$

$Z^{\prime} \quad$ Real part of complex impedance $(\Omega)$

$Z^{\prime \prime} \quad$ Imaginary part of complex impedance $(\Omega)$

\section{Greek letters}

$\Delta I \quad$ Galvanostatic perturbation amplitude $(A)$

$\Delta U_{k} \quad$ k-th component of the output signal generated by the response of the system $(V)$

$\Delta U_{n k} \quad$ k-th component of the output signal associated to noise $(V)$

$\vartheta \quad$ Frequency domain independent variable $(\mathrm{Hz})$

$\chi^{2} \quad$ Fitting sum of squares parameter $\left(\Omega^{2}\right)$

$\varphi_{k} \quad$ Offset of the k-th component of the output signal ( $\mathrm{rad}$ )

$\omega \quad$ Angular frequency $\left(\mathrm{rad} \cdot \mathrm{s}^{-1}\right)$ 


\section{Acknowledgments}

The authors are very grateful to the Generalitat Valenciana for its economic support in form of Vali+d grant (Ref: ACIF-2013-268). 


\section{References}

[1] M.E. Orazem, B. Tribollet, Electrochemical impedance spectroscopy, John Wiley \& Sons, New Jersey, 2008.

[2] E. Barsoukov, J.R. Macdonald, Impedance spectroscopy. Theory, experiment and applications, John Wiley \& Sons, New Jersey, 2005.

[3] D.D. Macdonald, Reflections on the history of electrochemical impedance spectroscopy, Electrochim. Acta, 51 (2006) 1376

[4] F. Ciucci, C. Chen, Analysis of electrochemica impedance spectrosocpy data using the distribution of relaxation times: A Bayesian and hierarchical Bayesian approach, Electrochim. Acta, 167 (2015) 439.

[5] Y. Fu et al., Heterogeneous electrocatalysis in porous cathodes of solid oxide fuel cells, Electrochim. Acta, 159 (2015) 71.

[6] D. Vladikova et al., Impedance spectroscopy studies of dual membrane fuel cell, Electrochim. Acta, 56 (2011) 7955.

[7] M. Lang, C. Auer, A. Eismann, P. Szabo, N. Wagner, Investigation of solid oxide fuel cell short stacks for mobile applications by electrochemical impedance spectroscopy, Electrochim. Acta, 53 (2008) 7509.

[8] M. Mamlouk, K. Scott, Analysis of high temperature polymer electrolyte membrane fuel cell electrodes using electrochemical impedance spectroscopy, Electrochim. Acta, 56 (2011) 5493.

[9] C. Boyer, S. Gamburzev, O. Velev, S. Srinivasan, A.J. Appleby, Measurement of proton conductivity in the active layer of PEM fuel cell gas diffusion electrodes, Electrochim. Acta, 43 (1998) 3703.

[10] O. Reid, F.S., Easton, E.B. Saleh, Determining electrichemically active surface area in PEM fuel cell electrodes with electrochemical impedance spectroscopy and its aplication to catalyst durability, Electrochim. Acta, 114 (2013) 278.

[11] S.S. Zhang, K. Xu, T.R. Jow, EIS study on the formation of solid electrolyte interface in Li-ion battery, Electrochim. Acta, 51 (2006) 1636. 
[12] J. Yun et al., In-situ electrochemical coating of Ag nanoparticles onto graphite electrode with enhanced performance for Li-on batteries, Electrochim. Acta, 155 (2015) 396.

[13] A.C. Ciubotariu, L. Benea, M., Dragan, V. Lakatos-Varsanyl, Electrochemical impedance spectroscopy and corrosion behaviour of Al2O3-Ni nano composite coatings, Electrochim. Acta, 53 (2008) 4557.

[14] J. Molina et al., Electrochemical characterization of electrochemically reduced graphene coatings on platinum. Electrochemical study of dye adsorption, Electrochim. Acta, 166 (2015) 54.

[15] E.A Franceschini, G.I. Lacconi, H.R Corti, Kinetics of the hydrogen evolution on nickel in alkaline solution: new insight from rotating disk electrode and impedance spectroscopy, Electrochim. Acta, 159 (2015) 210.

[16] B. Pejcic, R. De Marco, Impedance spectroscopy: Over 35 years of electrochemical sensor optimization, Electrochim. Acta, 51 (2006) 6217.

[17] L. Manjakkal et al., Electrochemical impedance spectroscopic analysis of RuO2 based thick film pH sensors, Electrochim. Acta, 168 (2915) 246.

[18] R. Pauliukaite, M.E. Ghica, O. Fatibello-Filho, C.M.A. Brett, Electrochemical impedance studies of chitosan-modified electrodes for application in electrochemical sensores and biosensors, Electrochim. Acta, 55 (2010) 6239.

[19] F. Long, Z. Zhang, J. Wang, Liang Yan, B. Zhou, Cobalt-nickel bimetalic nanoparticles decorated graphene sensitized imprinted electrochemical sensor for determination of octylphenol, Electrochim. Acta, 168 (2015) 337.

[20] J. Chen, Z. Xia, H. Li, Q. Li, Y. Zhang, Preparation of highly capacitive polyaniline/black TiO2 nanotubes as supercapcitor electrode by hidrogenation and electrochimical deposition, Electrochim. Acta, 166 (2015) 174.

[21] E. Karaca, N. Özçiçek, K. Pekmez, Galvanostatic deposition of polypyrrole in the presence of tartaric acid for electrochemical supercapacitor, Electrochim. Acta, 147 (2014) 545.

[22] Y. Zhang, Y. Mo, Preparation of $\mathrm{MnO} 2$ electrodes coated by Sb-doped SnO2 and their effect on electrochemical performance for supercapacitors, Electrochim. Acta, 142 (2014) 76. 
[23] C. Yadem, T. Repo, R. Silvennoinen, Dielectric relaxations in starch-water solutions, Electrochim. Acta, 171 (2015) 42.

[24] T. Vidakovic-Koch, V.K. Mittal, T.Q.N. Do, M. Varnicic, K. Sundmacher, Application of electrochemical impedance spectroscopy for studying of enzyme kinetics, Electrochim. Acta, 110 (2013) 94.

[25] R. Wang, J. Di, J. Ma, Z. Ma, Highly sensitive detection of cancer cells by electrochemical impedance spectroscopy, Electrochim. Acta, 61 (2012) 179.

[26] M. Naumowicz, A.D. Petelska, Z.A. Figaszewski, Impedance spectroscopic onvestigation between phosphatidyethanolamine and alfa-tocopherol in bilayer membranes, Electrochim. Acta, 54 (2009) 1089.

[27] K. Mallaiya, S. Rameshkumar, S.S. Subramanian, S. Ramalingam, T. Ramachandran, Electrochemical impedance studies on the interation of midazolam with planar lipid bilayer, Electrochim. Acta, 138 (2014) 360.

[28] W.M. Hassen, V. Duplan, E. Frost, J.J. Dubowski, Quantification of influenza A virus in the presence of extraneous protein using electrochemical impedance spectroscopy, Electrochim. Acta, 56 (2011) 8325.

[29] A. Sargent, O.A. Sadik, Monitoring antibody-antigen reactions at conducting polymer-based immunosensors using impedance spectroscopy, Electrochim. Acta, 44 (1999) 4667.

[30] M. Tulodziecki, J.M. Tarascon, P.L. Taberna, C. Guéry, Importance of the double layer structure in the electrochemical deposition of Co from soluble Co2+ based precursors in ionic liquid media, Electrochim. Acta, 134 (2014) 55.

[31] R.M. Torresi et al., Convection mass transport in ionic liquids studied by electrochemical and electrohydrodynamic impedance spectroscopy, Electrochim. Acta, 93 (2013) 32.

[32] W. Wang, A. Alfantazi, An electrochemical impedance spectroscopy and polarization study of the role of crystallographic orientation on electrochemical behavior of niobium, Electrochim. Acta, 131 (2014) 79. 
[33] B. Yan et al., Electrochemical impedance spectroscopy illuminating performance evolution of porous core-shell strcutured nickel/nickel oxide anode materials, Electrochim. Acta, 164 (2015) 55.

[34] D.D. Macdonald, E. Sikora Characterizing electrochemical systems in the frequency domain, Electrochim. Acta, 43 (1997) 87.

[35] M. Urquidi-Macdonald, S. Real, D.D. Macdonald, Applications of Kramers-Kronig transforms in the analysis of electrochemical impedance data- III. Stability and linearity, Electrochim. Acta, 35 (1990) 1559.

[36] W. Aperador, J.H. Bautista Ruíz, O. Pardo Cuervo, Electrochemical behaviour of thin films of $\mathrm{CrN} / \mathrm{Cr}$ obtained varying the bias potential, Rev. Mex. Ing. Quim., 11 (2012) 145.

[37] S. Noyel Victoria, S. Ramanathan, Effect of potential drift and ac amplitude on the electrochemical impedance spectra, Electrochim. Acta, 56 (2011) 2606.

[38] K. Darowicki, Linearization in impedance measurements, Electrochim. Acta, 42 (1997) 1781.

[39] K. Darowicki, The amplitude analysis of impedance spectra, Electrochim. Acta, 40 (1995) 439.

[40] M. Kiel, O. Bohlen, D.U. Sauer, Harmonic analysis for identification of nonlinearities in impedance spectroscopy, Electrochim. Acta, 53 (2008) 7367.

[41] X. Yuan, C. Song, H. Wang, J. Zhang, Electrochemical impedance spectroscopy in PEM fuel cells. Fundamentals and applications, Springer, London, 2010.

[42] W. Lai, Fourier analysis of complex impedance (amplitude and phase) in nonlinear systems: A case study of diodes, Electrochim. Acta, 55 (2010) 5511.

[43] G.S. Popkirowv, R.N. Schindler, Effect of sample nonlinearity on the performance of time domain electrochemical impedance spectroscopy, Electrochim. Acta, 40 (1995) 2511.

[44] B. Hirschorn, B. Tribollet, M.E. Orazem, On selection of the perturbation amplitude required to avoid nonlinear effects in impedance measurements, Israel J. Chem., 48 (2008) 133. 
[45] J.P. Diard, B. Le Gorrec, C. Montella, Deviation from the polarization resistance due to non-linearity. I- Theoretical formulation, J. Electroanal. Chem., 432 (1997) 27.

[46] E. Van Gheem et al., Electrochemical impedance spectroscopy in the presence of non-linear distortions and non-stationary behaviour. Part I: theory and validation, Electrochim. Acta, 49 (2004) 4753.

[47] J. Arrillaga, N.R. Watson, S. Chaen, Power systems quality assessment, Wiley, New York, 2000.

[48] Asociación Española de Normalización y Certificación (AENOR), Voltage characteristics of electricity supplied by public electricity networks, UNE-EN 50160:2011, 2011.

[49] Institute of Electrical and Electronics Engineers (IEEE), IEEE recommended practice and requirements for harmonic control in electrical power systems, IEEE Std. 11001999, 1999.

[50] D. Shmilovitz, On the definition of total harmonic distortion and its effects on measurement interpretation, IEEE T. Power Deliver., 20 (2005) 526.

[51] I. Herraiz Cardona, Desarrollo de nuevos materiales de electrodo para la obtención de hidrógeno a partir de la electrolisis alcalina del agua, PhD thesis, Universidad Politècnica de València, Valencia (Spain), 2012.

[52] I. Herraiz-Cardona, E. Ortega, V. Pérez-Herranz, Impedance study of hydrogen evolution on $\mathrm{Ni} / \mathrm{Zn}$ and $\mathrm{Ni}-\mathrm{Co} / \mathrm{Zn}$ stainless steel based electrodeposits, Electrochim. Acta, 56, (2011) 1308.

[53] I. Herraiz-Cardona, E. Ortega, J. García Antón, V. Pérez-Herranz, Assessment of the roughness factor effect and the intrinsic catalytic activity for hydrogen evolution reaction on Ni-based electrodeposits, Int. J. Hydrogen Energ., 36 (2011) 9428.

[54] I. Herraiz-Cardona, E. Ortega, L. Vázquez-Gómez, V. Pérez-Herranz, Electrochemical characterization of a $\mathrm{NiCo} / \mathrm{Zn}$ cathode for hydrogen generation, Int. J. Hydrogen Energ., 36 (2011) 11578.

[55] J. García-Antón, A. Igual-Muñoz, J.L Guiñon, V. Pérez-Herranz, Horizontal cell for electro-optical analysis of electrochemical processes, ES Patent P-200002526, October 20,2000 . 
[56] B. Hirschorn, M.E. Orazem, On the sensitivity of the Kramers-Kronig relations to nonlinear effects in impedance measurements, J. Electrochem. Soc., 156 (2009) C354.

[57] T.P. Pavlic, Lissajous figures, ECE 209 documentation, The Ohio State University, Columbus, 2009.

[58] P. Agarwal, M.E. Orazem, Application of measurement models to impedance spectroscopy. III. Evaluation of consistency with the Kramers-Kronig relations, J. Electrochem. Soc., 142 (1995) 4159.

[59] Q. Mao, U. Krewer, R. Hanke-Rauschenbach, Total harmonic distortion analysis for direct methanol fuel cell anode, Electrochem. Commun., 12, (2010) 1517.

[60] Q. Mao, U. Krewer, R. Hanke-Rauschenbach, Sensing methanol concentration in direct methanol fuel cell with total harmonic distortion: Theory and application, Electrochim. Acta, 68, (2012) 60.

[61] Q. Mao, U. Krewer, R. Hanke-Rauschenbach, Total harmonic distortion analysis of oxygen reduction reaction in proton exchange membrane fuel cell, Electrochim. Acta, 103, (2013) 188. 
LIST OF TABLES.

Table 1. EIS measurement parameters 



\section{LIST OF FIGURES.}

Figure 1. Linearity assessment method

Figure 2. Experimental setup

Figure 3. Nyquist plots obtained for perturbation amplitudes of $0.1 \mathrm{~mA}, 2 \mathrm{~mA}, 6 \mathrm{~mA}$ and $10 \mathrm{~mA}$

Figure 4. AC signals for different excited frequencies for two different amplitudes (1 $\mathrm{mA}$ and $10 \mathrm{~mA}$ )

Figure 5. Lissajous figures for different excited frequencies for two different amplitudes (1 $\mathrm{mA}$ and $10 \mathrm{~mA}$ )

Figure 6. Perturbation signal $\mathcal{T H \mathcal { D }}$ curves for low perturbation amplitudes (a) and high perturbation amplitudes (b)

Figure 7. Response signal THD curves for low perturbation amplitudes (a) and high perturbation amplitudes (b)

Figure 8. Critical parameter curve for the perturbation signal

Figure 9. Critical parameter curve for the response signal

Figure 10. Input and output signals

Figure 11. Model fitting to the linear zone of the experimental critical parameter curve

Figure 12. Nonlinear component of the critical parameter for each perturbation amplitude 
Table 1. EIS measurement parameters

Measurement parameter

Value

\begin{tabular}{|c|c|}
\hline Integration time & $1.0 \mathrm{~s}$ \\
\hline Number of integration cycles & 1 cycle \\
\hline Number of stabilization cycles & 10 cycles \\
\hline Number of stabilization cycles & $3.0 \mathrm{~s}$ \\
\hline Minimum stabilization cycle & 0.00 \\
\hline
\end{tabular}




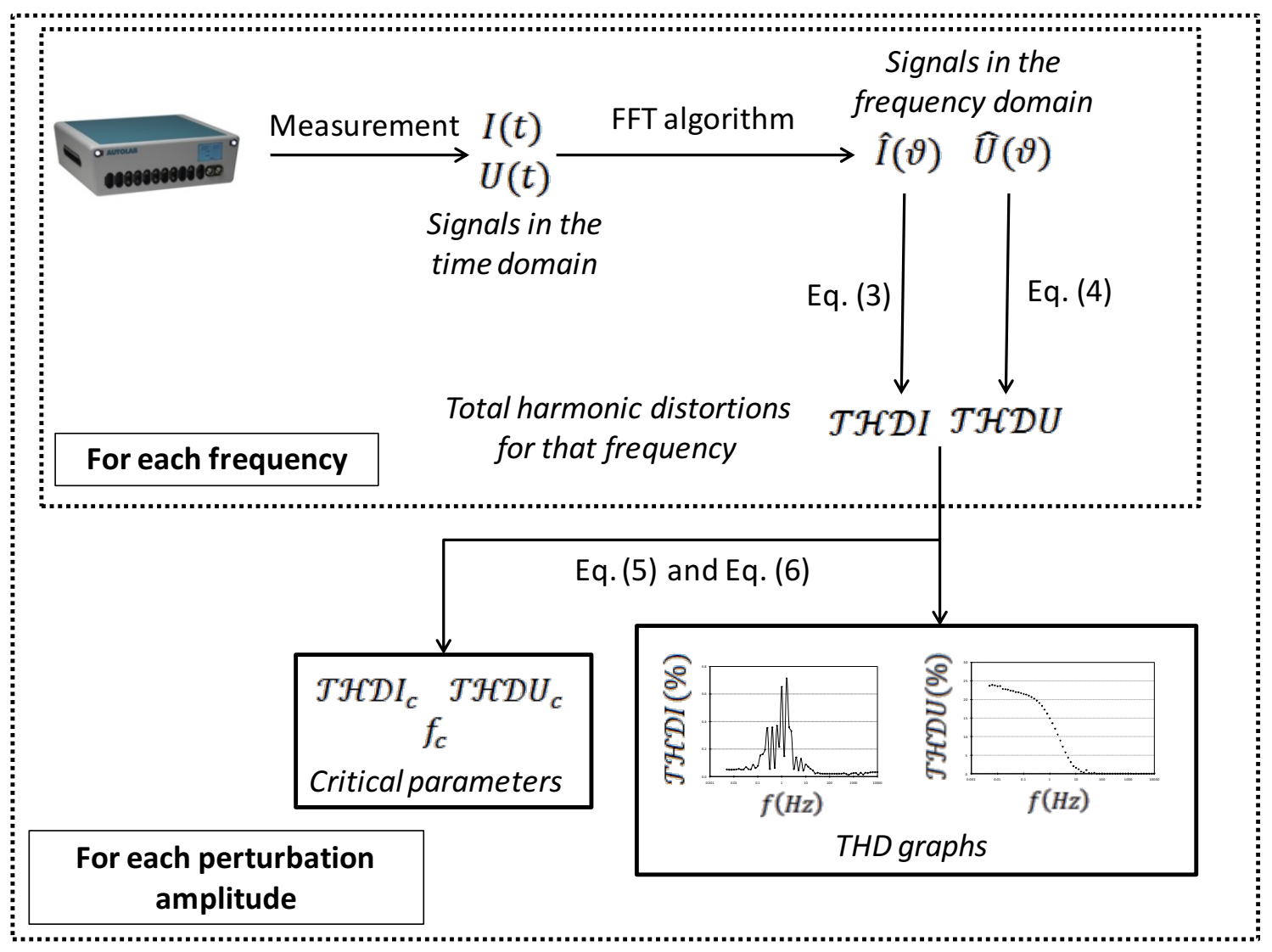

Figure 1. Linearity assessment method 


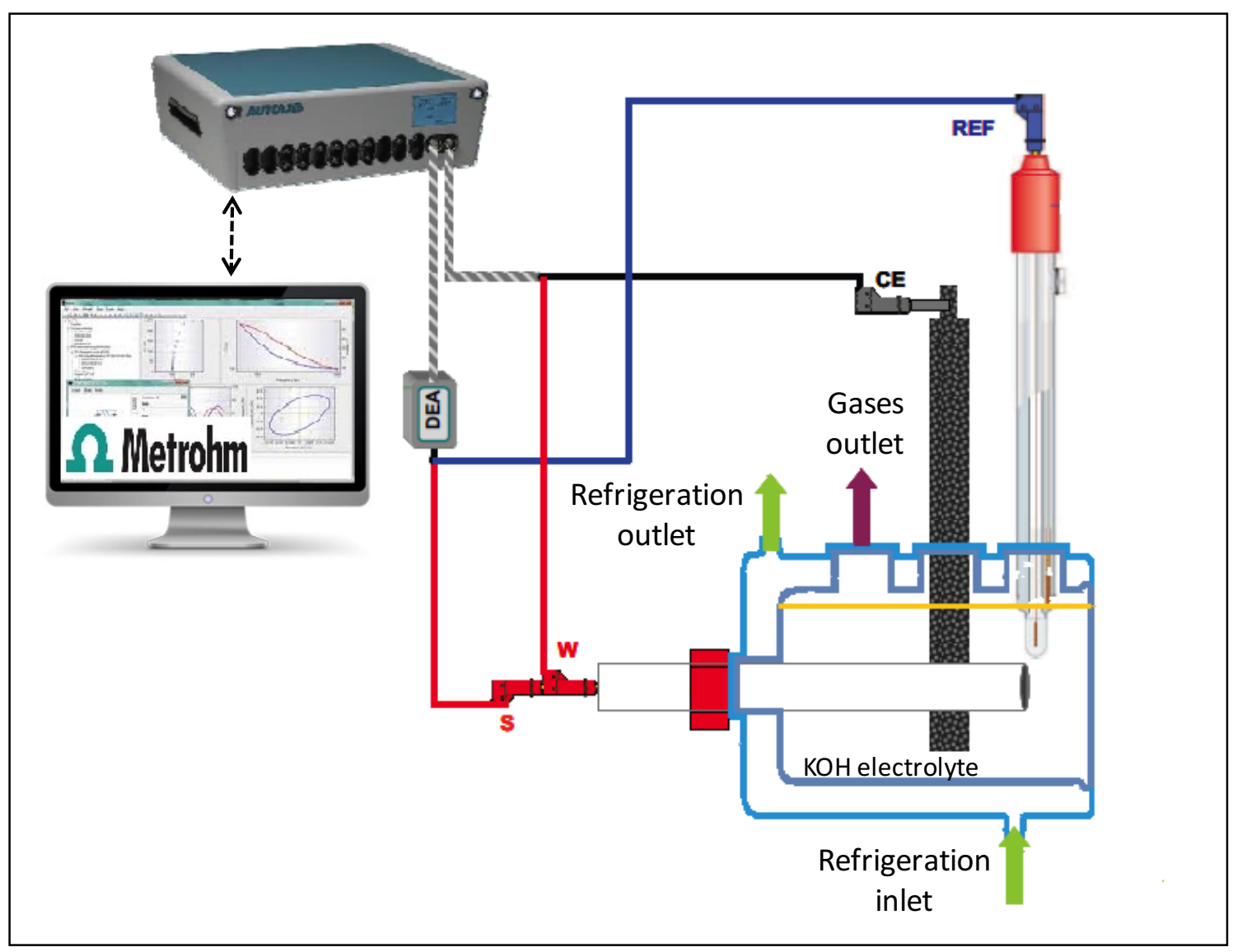

Figure 2. Experimental setup 




Figure 3. Nyquist plots obtained for perturbation amplitudes of $0.1 \mathrm{~mA}, 2 \mathrm{~mA}, 6 \mathrm{~mA}$ and $10 \mathrm{~mA}$ 


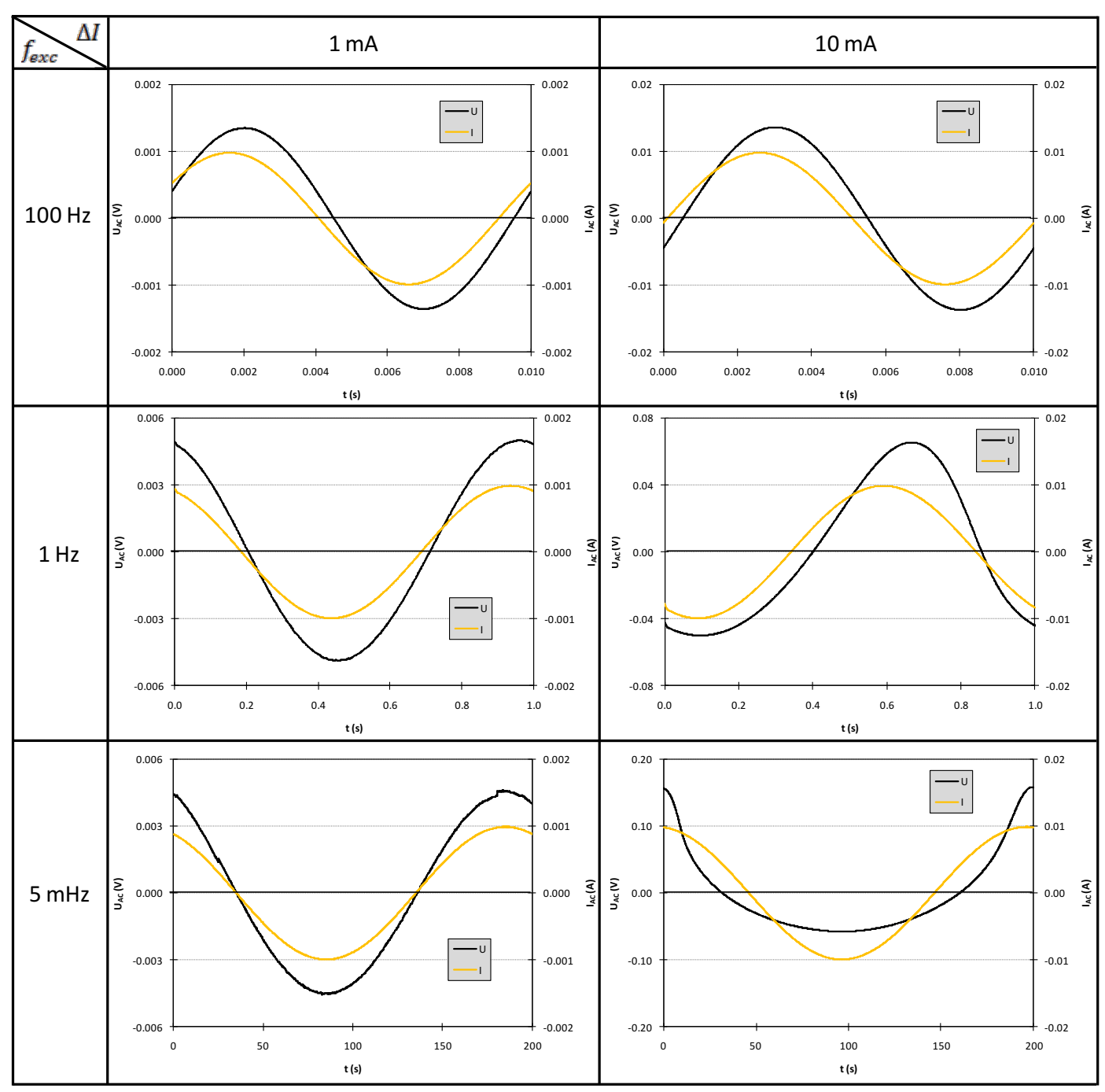

Figure 4. AC signals for different excited frequencies for two different amplitudes (1 $\mathrm{mA}$ and $10 \mathrm{~mA}$ ) 




Figure 5. Lissajous figures for different excited frequencies for two different amplitudes ( $1 \mathrm{~mA}$ and $10 \mathrm{~mA}$ ) 




b



Figure 6. Perturbation signal $\boldsymbol{T H \mathcal { D }}$ curves for low perturbation amplitudes (a) and high perturbation amplitudes (b) 




b

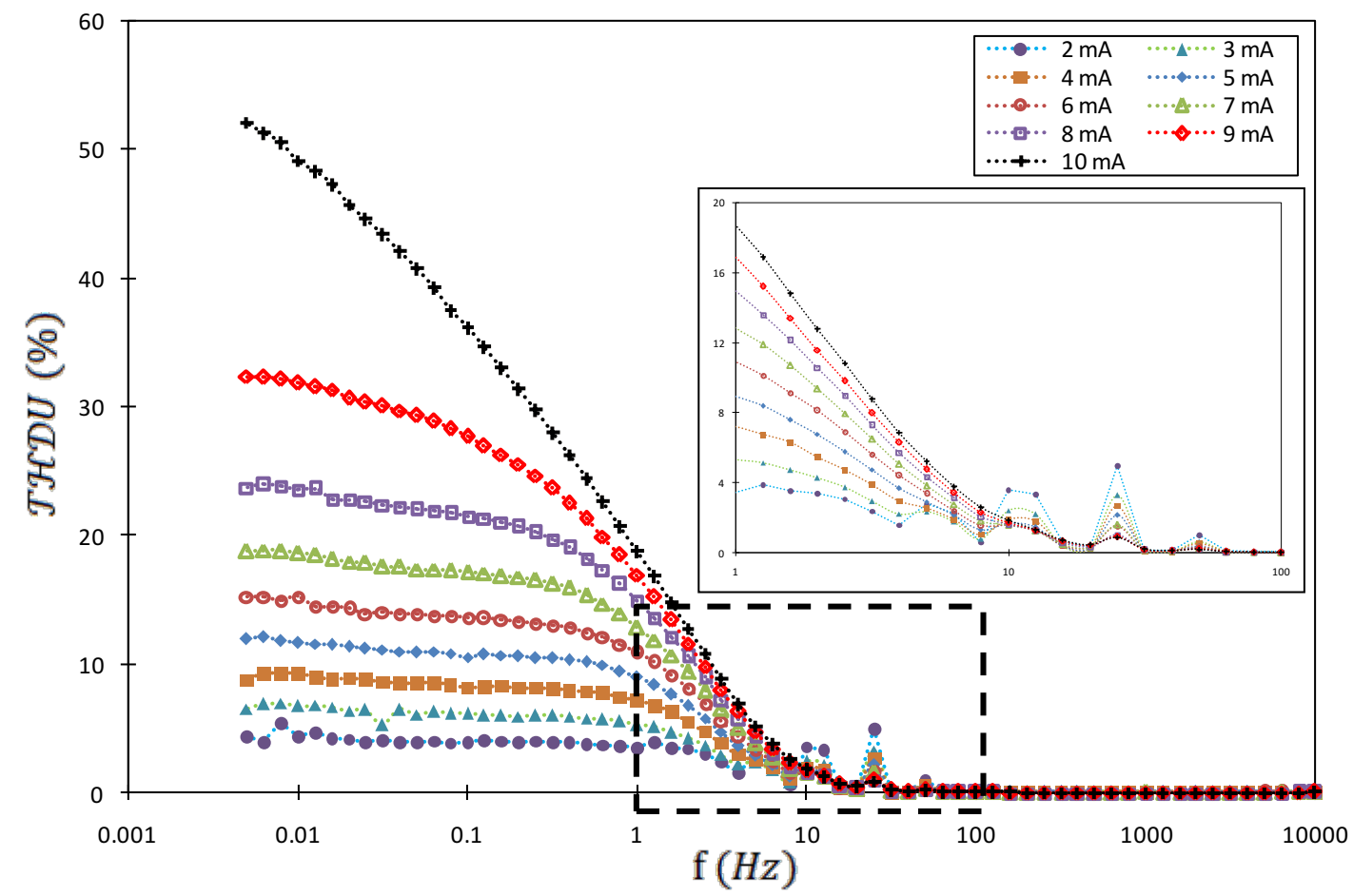

Figure 7. Response signal THD curves for low perturbation amplitudes (a) and high perturbation amplitudes (b) 


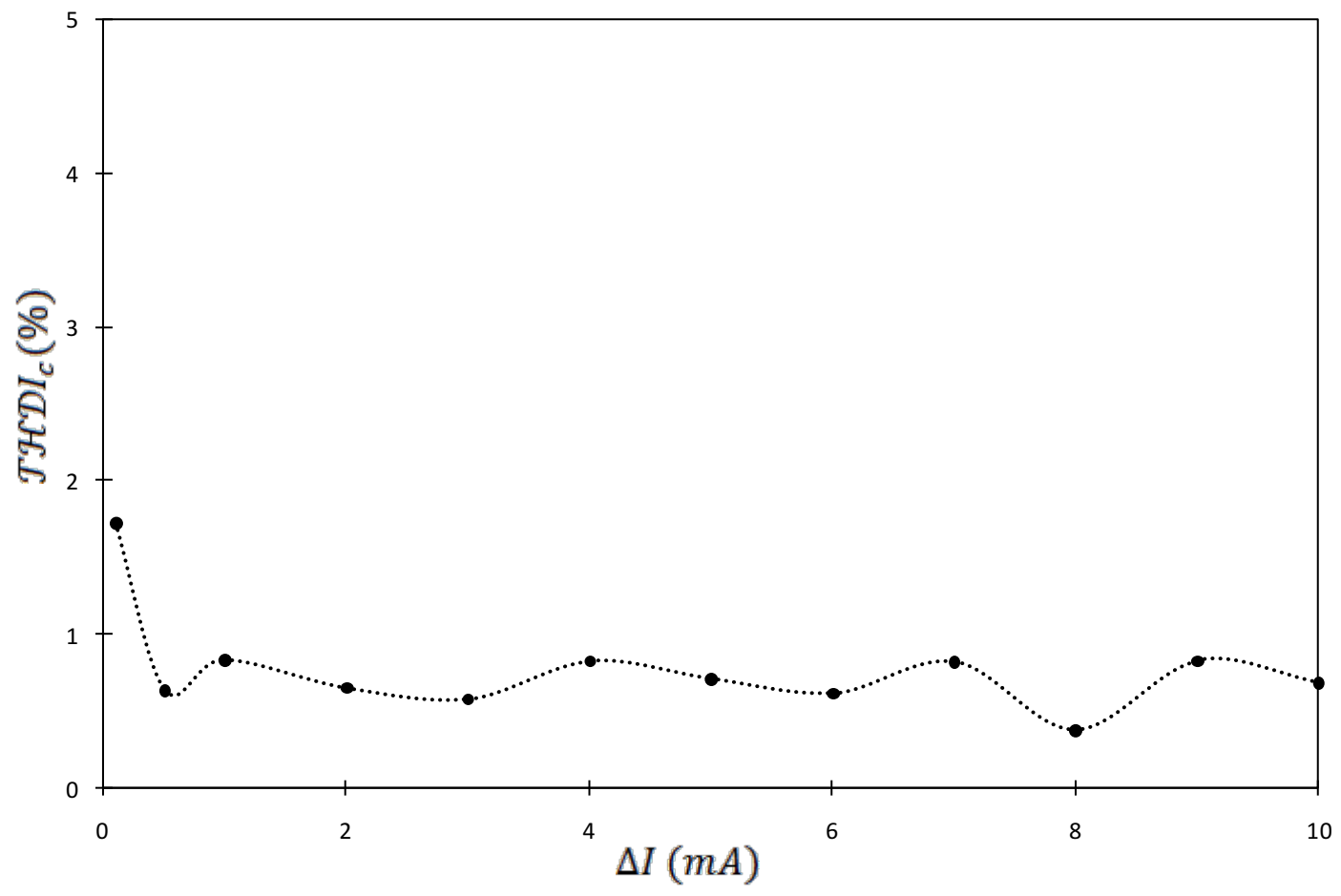

Figure 8. Critical parameter curve for the perturbation signal 


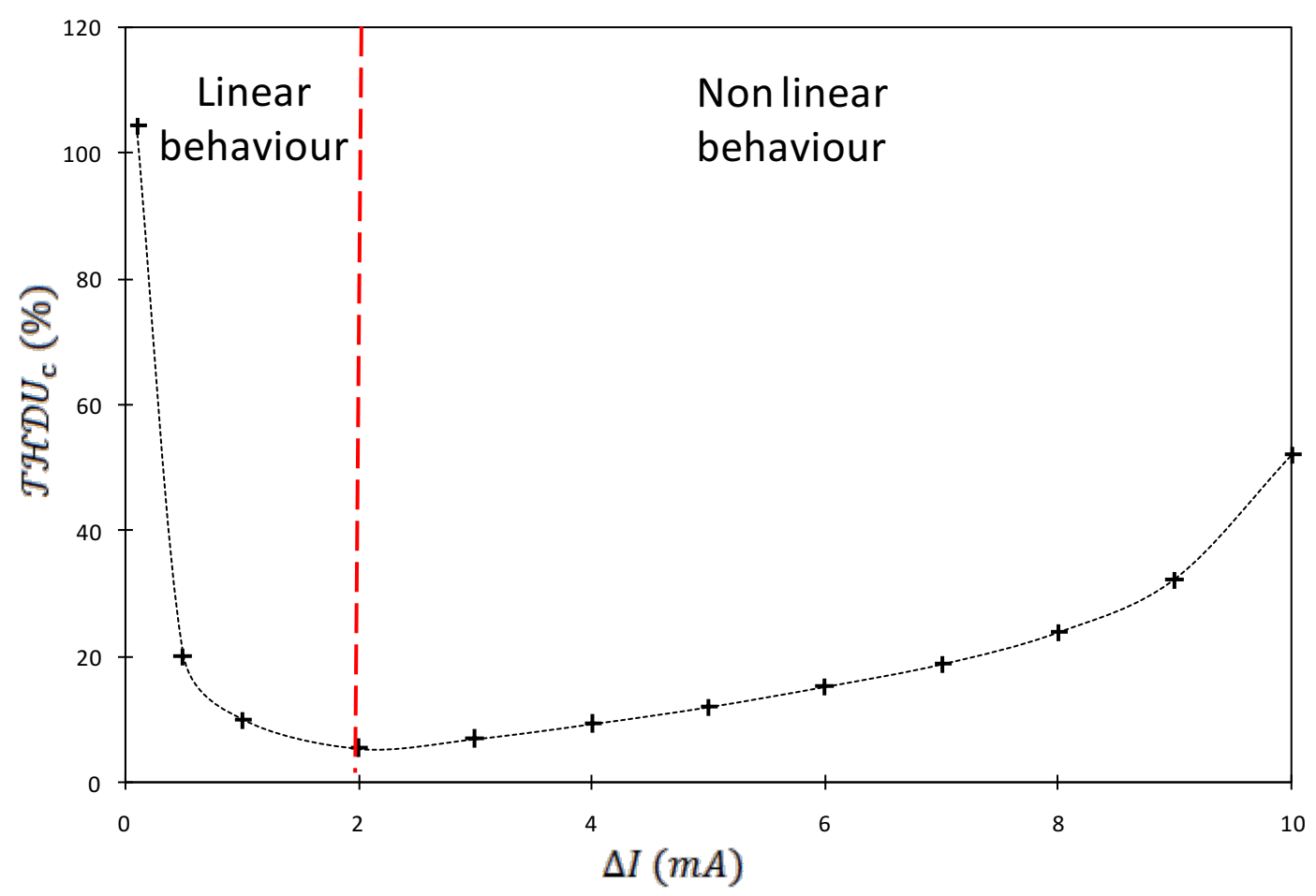

Figure 9. Critical parameter curve for the response signal 




Figure 10. Input and output signals 


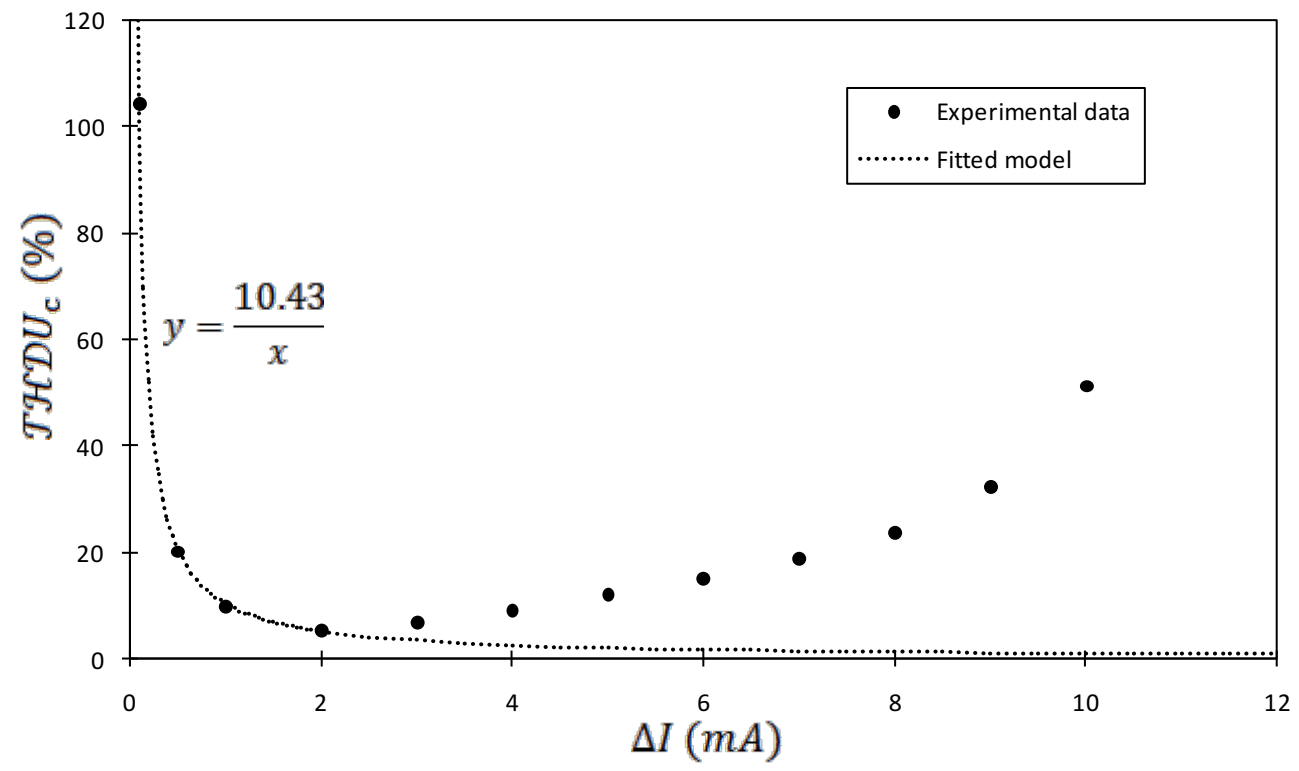

Figure 11. Model fitting to the linear zone of the experimental critical parameter curve 




Figure 12. Nonlinear component of the critical parameter for each perturbation amplitude 